\title{
The formation and dynamics of a blob on free and wall sheets induced by a drop impact on surfaces
}

\author{
Alexander I. Fedorchenko ${ }^{\text {a) }}$ and An-Bang Wang ${ }^{\text {b) }}$ \\ Institute of Applied Mechanics, National Taiwan University, No. 1, Roosevelt Road, Section 4, Taipei 106, \\ Taiwan, Republic of China
}

(Received 6 October 2003; accepted 27 July 2004; published online 5 October 2004)

\begin{abstract}
A physical model for describing an inviscid motion of free and wall liquid sheets induced by drop impact is presented. This model takes into account the formation of thick borders at the edge of a spreading drop, and the influence of advancing and receding contact angles on the dynamics of blob formation and motion. It has been shown that the blob motion on a free liquid sheet is described by a universal relationship (independent of the Weber number) in terms of dimensionless blob coordinates at both advancing and receding stages. For the case of blob formation and motion on a wall sheet at high Weber numbers, only the advancing stage is described by a universal relationship. The receding stage depends on the ratio of advancing and receding contact angles, but not on the Weber number. At the instant when the drop is at its maximum extension, the blob speed becomes equal to the liquid sheet velocity and the total kinetic energy of the drop is greater than zero. It is shown that the ratio of the instantaneous capillary wavelength to the sheet thickness is a constant 2.619 for a free sheet, when the capillary wave propagates away from the blob. (C) 2004 American Institute of Physics. [DOI: 10.1063/1.1793071]
\end{abstract}

\section{INTRODUCTION}

An important class of the free boundary flows is the flow in the thin sheets of liquid with free edges. The formation of free edges in such sheets is sometimes natural, as in flat or annular nozzles used in many atomizers (see, e.g., Lefebvre $^{1}$ ), and at drop impact on liquid or solid surfaces (see, e.g., Worthington, ${ }^{2,3}$ Fukai et al., ${ }^{4,5}$ Solonenko et al. ${ }^{6}$ ), or it may occur intentionally by the rupture of thin liquid films (see, e.g., Ranz, ${ }^{7}$ Pandit and Davidson ${ }^{8}$ ). The analysis of experimental evidences shows that the dynamics of the liquid flow is similar in all mentioned cases and is determined by thick borders (blob) formed at free edges. Due to the action of capillary forces blob moves upstream and enlarges with time, consuming the mass and momentum of liquid sheets. Usually liquid sheets disintegrate into droplets and the initial breakup takes place at a blob. The blob ejects filaments that then break up into secondary drops by capillary instability. Closeup views of the secondary droplets shedding from the rim that is moving around a spherical liquid film which has been punctured by a needle ${ }^{8}$ and from free edges of liquid films induced by the drop impact on deep $^{3}$ and shallow liquid layers (see, e.g., Yarin and Weiss, ${ }^{9}$ Wang and $\mathrm{Chen}^{10}$ ) show their intrinsic similarity. The blob formed at the edge of liquid sheet results in the capillary waves that propagate along both free and wall sheets, away from the blob (see Worthington, ${ }^{3}$ Aziz and $C$ Chandra ${ }^{11}$ ). A large number of authors carried out experimental and numerical studies of a free edge motion of a liquid sheet (see,

\footnotetext{
${ }^{a)}$ Permanent address: S. S. Kutateladze Institute of Thermophysics, Siberian Branch, Russian Academy of Sciences, 1 Acad. Lavrentyev Avenue, 630090 Novosibirsk, Russia.

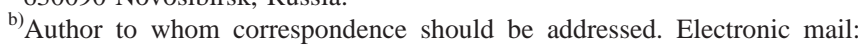
abwang@spring.iam.ntu.edu.tw
}

e.g., Ranz, ${ }^{7}$ Pandit and Davidson, ${ }^{8}$ Rayleigh, ${ }^{12}$ Taylor, ${ }^{13}$ Rosensweig, ${ }^{14}$ Oguz and Prosperetti ${ }^{15}$ ). Using the energy balance suggested by Rayleigh, ${ }^{12}$ Ranz $^{7}$ derived the following formula for the blob speed $U_{b}$ in the case when a needle punctured a soap bubble:

$$
U_{b}=\left(\frac{4 \sigma}{(\rho h)}\right)^{1 / 2},
$$

where $\sigma$ is the surface tension, $\rho$ is the liquid density, and $h$ is the thickness of the original soap film. Pandit and Davidson, ${ }^{8}$ Taylor, ${ }^{13}$ and Rosensweig ${ }^{14}$ used the momentum balance to derive the velocity of blobs forming at the edges of free flat sheets and soap bubbles and obtained the following formula:

$$
U_{b}=\left(\frac{2 \sigma}{\rho h}\right)^{1 / 2} .
$$

Both formulas give a constant blob velocity for films with the constant thickness. Culick ${ }^{16}$ noticed that the difference between Eqs. (1) and (2) is stipulated by ignoring the energy dissipation due to collision of the moving blob with elements of the stationary film.

Song and Tryggvason ${ }^{17}$ carried out an investigation of how a free two-dimensional edge recedes and accumulates fluid by solving the unsteady Navier-Stokes equations numerically for an initially stationary sheet. This study has shown that in the limits of high and low Ohnesorge numbers $\left[\mathrm{Oh}=\mu /(\rho \sigma h)^{1 / 2}, \mu\right.$ is the viscosity of the fluid], the receding speed of the edge is independent of $\mathrm{Oh}$ and it scales as $U_{b}$ $\sim[\sigma /(\rho h)]^{1 / 2}$ in the low $\mathrm{Oh}(\mathrm{Oh}<0.1)$ limit as proposed by Taylor. ${ }^{13}$ It should be noted that aforementioned theoretical investigations considered a stationary free liquid sheet of constant thickness. In cases of the blob formation at the 
edges of the free and wall sheets induced by drop impact on liquid and solid surfaces the velocity and thickness of the sheets are functions of time and space. In general cases they are unknown and have to be found.

A classical approach in solving the problem of drop impact on solid surface (see, e.g., Madejski, ${ }^{18}$ PasandidechFard et al. ${ }^{19}$ ) is based on the energy balance that equates initial drop kinetic energy to change in surface energy due to drop deformation and work against viscous forces. It is also assumed that at the instant $t_{\mathrm{m}}$ when the splat radius reaches its maximum the kinetic energy of the drop vanishes. In such an approach the influence of the blob on the drop spreading is neglected. However, Lugscheider et $_{\text {al. }}{ }^{20}$ have shown that this influence is important for prediction of the maximum splat radius at drop impact on a solid surface. Namely, the splat radius reaches a maximum when the speed of the drop edge $U_{\mathrm{de}}$ turns to zero. Since at the advancing stage of the drop spreading, $U_{\mathrm{de}}$ is equal to the difference between the velocity of the liquid $U_{s}$ and the speed of blob relative to the stationary liquid, $U_{b}$, from the condition $U_{\mathrm{de}}=0$ it follows that $U_{s}=U_{b}$ at $t=t_{m}$. It is evident from Eq. (2) that the velocity $U_{b}$ can reach large values for small $h$, therefore the kinetic energy of drop remains greater than zero at $t=t_{m}$. Thus, the blob is an important factor affecting the drop spreading and has to be taken into account. It is worth noting that numerical solution of the Navier-Stokes equations with account for the presence of surface tension performed by Fukai et $a .^{5}$ reveals the occurrence of drop recoiling and mass accumulation around the spreading drop periphery.

To concentrate on the influence of the blob itself on the dynamics of drop spreading, we neglect the effects of viscosity in this study. Starting from this point and following the approach, ${ }^{20-22}$ we consider the late stage of drop spreading at the beginning of which the drop assumes the disk-like shape with height $h_{0}$ and diameter $d_{0}$, and $h_{0} / d_{0} \ll 1$. The small parameter $\varepsilon=h_{0} / d_{0}$ allows us to construct the asymptotic expansion of the Euler equations under the corresponding boundary conditions. The derived equations are used to develop a mathematical and physical model for the motion and formation of a blob at the edges of thin free and wall sheet resulting from a drop impact.

\section{MATHEMATICAL MODEL OF THE LATE STAGE OF THE DROP SPREADING}

Let us consider a drop of diameter $D_{0}$ and velocity $U_{0}$ impinging perpendicularly on a flat rigid surface. At the instant $t=t_{0}$ which hereinafter is taken as the initial time (i.e., $t \rightarrow t-t_{0}$ ) for the late stage of the drop spreading, the drop assumes the disk-like shape with the height $h_{0}$ and the contact diameter $d_{0}$ as shown in Fig. 1 . The ratio $h_{0} / d_{0}$ is much less than unity at this time instant. Since the dynamics of spreading is characterized primarily by the Weber (We $\left.=\rho U_{0}^{2} D_{0} / \sigma\right)$ and Reynolds $\left(\operatorname{Re}=\rho U_{0} D_{0} / \mu\right)$ numbers, the aforementioned assumption for the drop spreading to be inviscid can be obtained on basis of analysis of Navier-Stokes equations and corresponding kinematic and dynamic boundary conditions in the dimensionless form and written as Re $\gg \mathrm{We} \gg 1 .{ }^{23}$ In that case the drop spreading is primarily in-

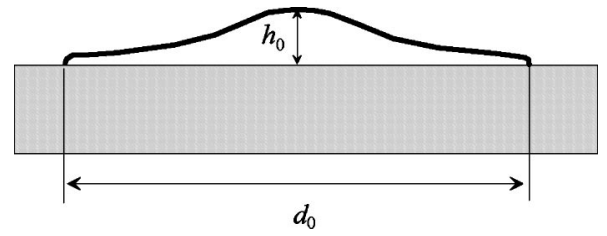

FIG. 1. Sketch of a drop at the beginning of the late stage of drop spreading; $d_{0}$ and $h_{0}\left(h_{0} / d_{0} \ll 1\right)$ are the initial contact diameter and the drop height, respectively.

ertia dominated. It is important to bear in mind that we restrict our consideration in the fast phase of the drop spreading. When the fast phase of the drop spreading with the velocity of the order of $U_{0}$ changes into the slow phase while the drop approaches its equilibrium shape determined by the static equilibrium contact angle $\theta_{e}$, the instantaneous contact line velocity $u_{c}$ is determined by the Hoffman-Voinov law: ${ }^{24-26} \mu u_{c} / \sigma \approx k\left(\theta^{3}-\theta_{e}^{3}\right)$, where $k \approx$ const and $\theta$ is the instantaneous dynamic contact angle. The viscosity plays an important role and has to be taken into account for the latter case.

Accounting for above-mentioned reason, we assume that the drop collision with the surface results in incompressible, inviscid flow with the velocity potential $\varphi(x, y, z)$ beginning from the time instant $t=0$. Then the continuity and momentum equations take the forms

$$
\begin{aligned}
& \Delta \varphi+\partial_{z z} \varphi=0, \\
& \partial_{t} \varphi+(\nabla \varphi)^{2} / 2+\left(\partial_{z} \varphi\right)^{2} / 2=\left(p_{0}-p\right) / \rho,
\end{aligned}
$$

where $\boldsymbol{\nabla}=\left(\partial_{x}, \partial_{y}\right)$ and $\Delta=\partial_{x x}+\partial_{y y}$.

At the substrate $z=0$ the impermeability condition is imposed,

$$
\partial_{z} \varphi=0 .
$$

At the free surface $z=h(\mathbf{x}, t)$ we impose the kinematic condition

$$
\partial_{t} h+\nabla \varphi \cdot \nabla h=\partial_{z} \varphi
$$

and the dynamic condition accounting for capillary force

$$
p_{0}-p=\sigma \nabla \cdot\left[\nabla h /\left[1+(\nabla h)^{2}\right]^{1 / 2}\right] .
$$

Here $\mathbf{x}=(x, y)$ and $p_{0}$ is the ambient medium pressure.

Notice that the function $z=h(\mathbf{x}, t)$ describes the free boundary of the spreading drop except for the immediate vicinity of the moving solid-liquid-air contact line.

Let us introduce the following dimensionless variables: $\Phi=\varphi /\left(U_{0} d_{0}\right), T=U_{0} t / d_{0}, \mathbf{X}=\mathbf{x} / d_{0}, Z=z / h_{0}, P=p /\left(\rho U_{0}^{2}\right)$, and $H=h / h_{0}$. Then Eqs. (3)-(7) take the form

$$
\begin{aligned}
& \varepsilon^{2} \Delta \Phi+\partial_{Z Z} \Phi=0, \\
& \partial_{T} \Phi+\frac{(\nabla \Phi)^{2}}{2}+\varepsilon^{-2}\left(\partial_{Z} \Phi\right)^{2}=\frac{\varepsilon}{W_{0}} \nabla \cdot \frac{\boldsymbol{\nabla} H}{\left[1+\varepsilon^{2}(\nabla H)^{2}\right]^{1 / 2}},
\end{aligned}
$$

$\varepsilon^{2}\left(\partial_{T} H+\nabla \Phi \cdot \nabla H\right)=\partial_{Z} \Phi, Z=H$, 


$$
\partial_{Z} \Phi=0, Z=0,
$$

where $\mathrm{W}_{0}=\rho U_{0}^{2} d_{0} / \sigma$.

When deriving Eq. (9), we replaced the term on the right-hand side of Eq. (4) by Eq. (7).

The solution of Eqs. (8)-(11) we seek in the form of an asymptotic expansion of the function $\Phi(Z, \mathbf{X}, T)$ into a series of $\varepsilon Z$-powers:

$$
\Phi(Z, \mathbf{X}, T)=\sum_{n=0}^{\infty}(\varepsilon Z)^{2 n} f_{n}(\mathbf{X}, T) .
$$

Substituting Eq. (12) into Eq. (8) and equalizing the terms with the same powers of $\varepsilon$, we obtain a recurrent relationship for functions $f_{n}(\mathbf{X}, T)$, which is

$$
f_{n}=-\frac{\Delta f_{n-1}}{2 n(2 n-1)} \text {. }
$$

Rewriting Eq. (12) in terms of the formula Eq. (13) gives

$$
\Phi(Z, \mathbf{X}, T)=\sum_{n=0}^{\infty}(-1)^{n} \frac{(\varepsilon Z)^{2 n}}{(2 n) !} \Delta^{n} F(\mathbf{X}, T),
$$

where $F(\mathbf{X}, T)$ stands for $f_{0}(\mathbf{X}, T)$.

Substituting Eq. (14) into Eqs. (9) and (10), expanding for small $\varepsilon$, and holding the principal terms in both expansions, we have

$$
\begin{aligned}
& \partial_{T} F+\frac{(\nabla F)^{2}}{2}=\frac{\varepsilon}{W_{0}} \Delta H, \\
& \partial_{T} H+\nabla \cdot(H \nabla F)=0 .
\end{aligned}
$$

In Eq. (15) we hold the term with a small parameter $\varepsilon / \mathrm{W}_{0}$ multiplying by the highest derivative responsible for capillary effects. It is natural to expect that the effect of this term is mainly localized near the edge of a spreading drop, whereas its influence is insignificant for the main flow. Disregarding the right-hand side of Eq. (15), returning to dimension variables, and taking the gradient of Eq. (15), we obtain the final set of differential equations governing the motion of a fluid in a plane approximation, which describes the late stage of drop spreading (thin sheet flow approximation):

$$
\begin{aligned}
& \partial_{t} h+\nabla \cdot(h \mathbf{u})=0, \\
& \partial_{t} \mathbf{u}+(\mathbf{u} \cdot \boldsymbol{\nabla}) \mathbf{u}=0 .
\end{aligned}
$$

It should be noted that similar equations were previously postulated $^{9}$ as a simplified quasi-one-dimensional approach averaging the flow velocity over the thickness of the thin layer.

The system of Eqs. (17) and (18), with above-mentioned assumptions, can be applied for axisymmetric spreading of a drop after collision with a surface.

Assuming that, at $t=0$, the drop height and the velocity distributions in a spreading drop are $h_{0}(r)$ and $u_{0}(r)$ and passing to the cylindrical coordinate system in Eqs. (17) and (18), we arrive at the initial value problem for hyperbolic

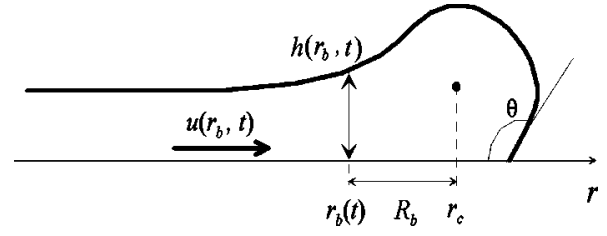

FIG. 2. Blob formation on the edge of spreading drop; $r_{c}$ and $R_{b}$ are the coordinates of the blob's center of mass and radius of the blob, respectively. $u\left(r_{b}, t\right)$ and $h\left(r_{b}, t\right)$ are the velocity and thickness of the wall sheet at the point with coordinate $r_{b}$ just in front of the blob, $\theta$ is the advancing contact angle.

system of equations, which describes the evolution of the drop's height and velocity at the late stage of a drop spreading,

$$
\begin{aligned}
& \partial_{t} u+u \partial_{r} u=0, \\
& \partial_{t} h r+\partial_{r} h u r=0,
\end{aligned}
$$

$$
u(r, 0)=u_{0}(r), h(r, 0)=h_{0}(r) .
$$
(21) is

The solution of Eq. (19) with the initial condition Eq.

$$
u=u_{0}(\xi), \quad r=\xi+u_{0}(\xi) t,
$$

where $\xi$ is the Lagrangian coordinate.

The solution of Eq. (20) takes the form

$$
h=\frac{\xi h_{0}(\xi)}{\left[\xi+u_{0}(\xi) t\right]\left[1+u_{0}^{\prime}(\xi) t\right]} \text {. }
$$

For later use the representation of $u_{0}(\xi)$ and $h_{0}(\xi)$ for small values of the Lagrangian coordinate $\xi$ will be needed, which reads

$$
u_{0}(\xi)=b \xi+O\left(\xi^{2}\right), h_{0}(\xi)=h_{00}+O(\xi)
$$

as $\xi \rightarrow 0$. Here $b=u_{0}^{\prime}(0)$ and $h_{00}$ are constants, whose exact values will be determined subsequently. Substituting Eq. (24) into Eqs. (22) and (23), we obtain

$$
u=r /\left(t+b^{-1}\right), h=h_{00} /(1+b t)^{2} .
$$

Introducing a new variable

$$
\tau=t+b^{-1},
$$

formulas in Eqs. (25) take the form

$$
u=r / \tau, h=h_{00} /(b \tau)^{2} .
$$

\section{MODEL FOR THE FORMATION AND MOVEMENT OF A BLOB ON THE EDGE OF A SPREADING DROP}

The edge of a spreading drop involves a moving contact line, which is the intersection of a liquid phase, ambient air, and a solid substrate. At the contact line the following boundary condition has to be satisfied: the angle between the tangent of the liquid-gas interface and solid surface is equal to the dynamic contact angle $\theta$ (see Fig. 2). Therefore, in the vicinity of the drop's edge, the effect of the capillary forces is significant. Since the shape of the spreading drop is far 
from that in equilibrium, capillary forces try to deform the leading edge of the drop resulting in the capillary wave propagating upstream. The characteristic propagation velocity scale can be estimated ${ }^{27}$ as the velocity of a capillary wave with the wave number $k=2 \pi / h_{00}$,

$$
C_{0}=\left[\sigma /\left(\rho h_{00}\right)\right]^{1 / 2}
$$

The ratio of $C_{0}$ to the initial drop impact velocity $U_{0}$ is

$$
C_{0} / U_{0}=\left(D_{0} / h_{00}\right)^{1 / 2} \mathrm{We}^{-1 / 2},
$$

and has a small value at high Weber numbers. As a result, the capillary waves cannot propagate upstream and they have been locked in the vicinity of the leading edge of the drop forming the blob. Therefore most part of the spreading drop remains undisturbed. Under the action of capillary forces the blob moves towards the spreading drop consuming the mass and momentum of the drop, so the consumed mass and momentum are determined by the time-varying height of the liquid film $h\left(r_{b}, t\right)$ and the velocity of liquid $u\left(r_{b}, t\right)$ just in front of the blob (see Fig. 2). Here $r_{b}$ is the coordinate of the edge of the spreading drop just before the blob, $r_{c}$ is the blob's center of mass, and $R_{b}=r_{c}-r_{b}$ is the blob radius.

Now let us derive the equations for the mass and momentum balance of the blob in a plane geometry. Later we will show that generalization for the case of the cylindrical coordinate system is rather simple. To find the relationship between the radius and mass of the blob let us assume that the cross section of the blob is the upper part of a circle with radius $R_{b}$ which intersects the solid surface at the point $A$ at the advancing contact angle $\theta$ (see Fig. 2). The cross-section area $S$ of the upper part of the circle is determined by the formula: $S=\theta R_{b}^{2}$. Consequently, the blob radius is

$$
R_{b}=(S / \theta)^{1 / 2}=[M /(\rho \theta)]^{1 / 2},
$$

where $M$ is the mass of the blob per unit length and $\rho$ is the liquid density.

Considering the blob as a moving body with a specific (i.e., per unit length) mass $M$ and momentum $M V$, where $V$ is the velocity of blob's center of mass, the changing of the blob mass and momentum is described by the following equations: balance of mass

$$
d M / d t=\rho h\left(r_{b}, t\right)\left[u\left(r_{b}, t\right)-d r_{b} / d t\right]
$$

balance of momentum

$$
\begin{aligned}
& d(M V) / d t=F_{u}+F_{\sigma}, \\
& F_{u}=\rho h\left(r_{b}, t\right) u\left(r_{b}, t\right)\left[u\left(r_{b}, t\right)-d r_{b} / d t\right],
\end{aligned}
$$

where $F_{u}$ is the specific momentum flux from the spreading drop into the blob and $F_{\sigma}$ is the radial component of resulting capillary forces applied to the blob. Let us determine the resulting vector of capillary forces $\mathbf{F}_{\sigma} d L$ acting on an element of the blob with length $d L$. This force could be found by integration of the excessive capillary pressure over the free surface $S$ of the blob (see Fig. 3)

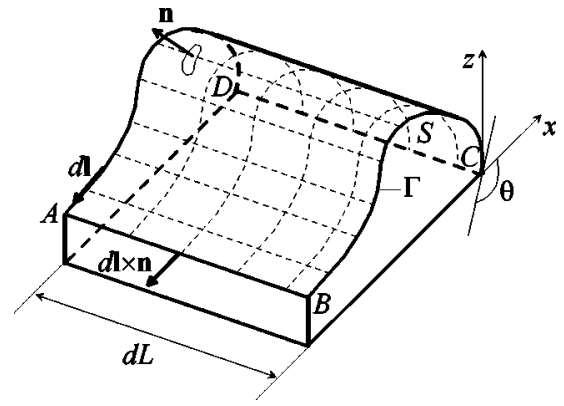

FIG. 3. Determination of the resulting vector of capillary forces applied to the blob.

$$
\mathbf{F}_{\sigma}=-\sigma \iint_{S} 2 H d \mathbf{S}
$$

Here $2 H$ is the average curvature of the free surface $d \mathbf{S}$ $=\mathbf{n} d S$, where $\mathbf{n}$ is an external normal to the element of free surface $d S$. Using formulas $2 H=\boldsymbol{\nabla} \cdot \mathbf{n}$ and $\mathbf{n}(\boldsymbol{\nabla} \cdot \mathbf{n})=-(\mathbf{n}$ $\times \boldsymbol{\nabla}) \times \mathbf{n}$, the Eq. (34) can be rewritten as

$$
\mathbf{F}_{\sigma}=\sigma \iint_{S}(d \mathbf{S} \times \nabla) \times \mathbf{n} .
$$

Applying Stokes's theorem to the surface integral in Eq. (35) yields

$$
\mathbf{F}_{\sigma}=\sigma \oint_{\Gamma} d \mathbf{l} \times \mathbf{n},
$$

where $\Gamma$ is the closed curve bounding the surface $S$. Contributions to the line integral Eq. (36) along curves $A D$ and $B C$ (see Fig. 3) annihilate each other, and contributions along curves $A B$ and $C D$ are easy to calculate taking into account that vector $d \mathbf{l} \times \mathbf{n}$ lies in the plane tangential to the surface $S$. Therefore, the projection of vector $\mathbf{F}_{\sigma}$ on $x$ direction takes the form

$$
F_{\sigma x}=-\sigma d L(1-\cos \theta) .
$$

Accounting for Eqs. (32), (33), and (37), we arrive at the following momentum equation for the blob

$$
\begin{aligned}
d(M V) / d t= & \rho h\left(r_{b}, t\right) u\left(r_{b}, t\right)\left[u\left(r_{b}, t\right)-d r_{b} / d t\right] \\
& -\sigma(1-\cos \theta) .
\end{aligned}
$$

Equations (31) and (38) have to be supplemented with a relation for the speed of the blob's center of mass $V$ $=d r_{c} / d t$. At the advancing stage of drop spreading, the change in the blob's mass is negligible. Consequently we can assume $r_{c} \approx r_{b}$. Then

$$
V=d r_{b} / d t \text {. }
$$

For the axisymmetric blob we can substitute $r_{b} M$ for $M$ in Eqs. (31) and (38) and multiply the right-hand sides of these equations by $r_{b}$ as long as $r_{b} \gg R_{b}$ :

$$
\frac{d\left(r_{b} M\right)}{d t}=\rho r_{b} h\left(r_{b}, t\right)\left[u\left(r_{b}, t\right)-\frac{d r_{b}}{d t}\right] \text {, }
$$




$$
\begin{aligned}
\frac{d\left(r_{b} M V\right)}{d t}= & \rho r_{b} h\left(r_{b}, t\right) u\left(r_{b}, t\right)\left[u\left(r_{b}, t\right)-\frac{d r_{b}}{d t}\right] \\
& -\sigma r_{b}(1-\cos \theta) .
\end{aligned}
$$

\section{ASYMPTOTIC APPROXIMATION FOR THE LATE STAGE OF DROP SPREADING}

Based on the aforesaid consideration, we can adopt the following pattern for the late stage of drop spreading: the drop impact creates a thin wall sheet with the blob at the edge. Under the action of capillary forces the blob moves towards the spreading drop, consuming the mass and momentum of the sheet. As the blob absorbs more and more liquid, the Lagrangian coordinate $\xi$ of inflowing liquid sheet elements tends to zero and the velocity and height profiles in the sheet tend to the solution Eq. (27), which is the limiting solution in the problem of spreading drop. When the blob speed becomes equal to the velocity of the liquid in the sheet, the blob stops. The blob coordinate $r_{b}$ at this moment is the maximum radius of the spreading drop. After that the blob starts moving towards the center of the drop and the drop begins to recede.

Since it is very difficult to measure precisely dynamic contact angle, we adopt the following approximation for $\theta$, as done in previous studies ${ }^{5,11,19,22}$ on a drop impact on a solid surface:

$$
\theta= \begin{cases}\theta_{1}=\text { const }_{1}, & d r_{b} / d t>0, \\ \theta_{2}=\text { const }_{2}, & d r_{b} / d t<0,\end{cases}
$$

where const $t_{1}$ and const ${ }_{2}$ are the assumed constant values for the dynamic contact angle at advancing and receding stages, respectively.

Using the Heaviside unit step function $H(x)$, Eq. (42) can be represented in the following form: $\theta=\theta_{1} H\left(d r_{b} / d t\right)$ $+\theta_{2} H\left(-d r_{b} / d t\right)$.

\section{A. Blob coordinates and governing equations for advancing/receding stage}

Thus, an asymptotic behavior of the system "spreading drop + blob" is described by the following system of equations:

$$
\begin{aligned}
& \frac{d m}{d \tau}=\rho r_{b} \frac{h_{00}}{b^{2} \tau^{2}}\left(\frac{r_{b}}{\tau}-\frac{d r_{b}}{d \tau}\right), \\
& \frac{d}{d \tau}\left(m \frac{d r_{b}}{d \tau}\right)=\rho \frac{r_{b}^{2} h_{00}}{b^{2} \tau^{3}}\left(\frac{r_{b}}{\tau}-\frac{d r_{b}}{d \tau}\right)-r_{b} \sigma(1-\cos \theta) .
\end{aligned}
$$

Here $m=r_{b} M$ and $\tau=t+b^{-1}$.

Equations (43) and (44) have to be supplemented by the following initial conditions:

$$
m=0, r_{b}=r_{b 0} \text { at } \tau=b^{-1},
$$

which mean that at the initial time the blob mass is zero and its coordinate is $r_{b 0}$. The third initial condition follows from Eqs. (43) and (44) accounting for the initial conditions, Eq. (45):

$$
\frac{d r_{b}}{d \tau}=\frac{r_{b 0}}{\tau}-\left[\frac{\sigma\left(1-\cos \theta_{a}\right)}{\rho h_{00}}\right]^{1 / 2} \text { at } \tau=b^{-1} .
$$

This means that the speed of the blob relative to the film is equal to $\left[\sigma\left(1-\cos \theta_{1}\right) /\left(\rho h_{00}\right)\right]^{1 / 2}$. If we apply Eq. (46) to the blob formation at the edge of free liquid sheet emerging, for example, at drop impact on a liquid layer, then the multiplier $(1-\cos \theta)$ has to be replaced by 2 because a surface tension acts on both sides of the liquid sheet and $\theta=90^{\circ}$. Formally, this case can be considered as the blob motion with $\theta$ $=180^{\circ}$, and Eq. (46) will describe the blob motion on both wall and free sheets. Thus, the blob speed on a stationary wall liquid sheet coincides, with an accuracy of multiplier, with the solution obtained by Taylor. ${ }^{13}$ Integrating Eq. (43) and accounting for Eq. (45), we obtain the expression for the blob mass as a function of time:

$$
m=0.5 \rho h_{00}\left[r_{b 0}^{2}-r_{b}^{2} /(b \tau)^{2}\right] .
$$

Substituting Eq. (45) into Eq. (42), we come to the differential equation for $r_{b}$,

$$
\frac{d^{2}}{d \tau^{2}} r_{b}\left[1-\frac{1}{3} r_{b}^{2} /\left(r_{b 0} b \tau\right)^{2}\right]+\frac{2 \sigma(1-\cos \theta) r_{b}}{\rho h_{00} r_{b 0}^{2}}=0 .
$$

The initial conditions for this equation are Eqs. (45) and (46).

Let us introduce dimensionless variables, hereinafter called "blob coordinates,"

$$
\begin{aligned}
& \bar{\tau}=\left[\frac{\sigma\left(1-\cos \theta_{1}\right)}{\rho h_{00}}\right]^{1 / 2} \frac{\tau}{r_{b 0}}, \\
& \bar{r}_{b}=\left[\frac{\sigma\left(1-\cos \theta_{1}\right)}{\rho h_{00}}\right]^{1 / 2} \frac{r_{b}}{b r_{b 0}{ }^{2}} .
\end{aligned}
$$

The convenience of using the blob coordinates will become obvious later.

By means of coordinates transformation expressed by Eqs. (49) and (50), Eqs. (45), (46), and (48) can be rewritten as follows: advancing stage

$$
\begin{aligned}
& \frac{d^{2}}{d \bar{\tau}^{2}} \bar{r}_{b}\left(1-\frac{\bar{r}_{b}^{2}}{3 \bar{\tau}^{2}}\right)+\omega_{a}^{2} \bar{r}_{b}=0, \\
& \bar{r}_{b}=\mathrm{We}_{*}{ }^{-1 / 2}, \frac{d \bar{r}_{b}}{d \bar{\tau}}=1-\mathrm{We}_{*}^{-1 / 2} \text { at } \bar{\tau}=\mathrm{We}_{*}^{-1 / 2} ;
\end{aligned}
$$

receding stage

$$
\begin{aligned}
& \frac{d^{2}}{d \bar{\tau}^{2}} \bar{r}_{b}\left(1-\frac{\bar{r}_{b}^{2}}{3 \bar{\tau}^{2}}\right)+\omega_{r}^{2} \bar{r}_{b}=0, \\
& \bar{r}_{b}=\bar{r}_{b}\left(\bar{\tau}_{\max }\right), \frac{d \bar{r}_{b}}{d \bar{\tau}}=0 \text { at } \bar{\tau}=\bar{\tau}_{\max },
\end{aligned}
$$

where $\mathrm{We}_{*}=\rho b^{2} r_{b 0}{ }^{2} h_{00} /\left[\sigma\left(1-\cos \theta_{1}\right)\right], \omega_{a}=2^{1 / 2}, \omega_{r}=[2(1$ $\left.\left.-\cos \theta_{2}\right) /\left(1-\cos \theta_{1}\right)\right]^{1 / 2}, \bar{\tau}_{\max }$ is the instant when the coordinate of the blob reaches its maximum value $\bar{r}_{b \max }=\bar{r}_{b}\left(\bar{\tau}_{\max }\right)$ at the advancing stage. For the case of blob formation at the edge of a free liquid sheet, when there is no contact angle hysteresis, both advancing and receding stages are described 
by Eq. (51) and initial boundary conditions by Eq. (52).

By equating the drop volumes and kinetic energies before and after impact, the parameters $b, r_{b 0}$, and $h_{00}$ can be expressed through the initial velocity of impact $U_{0}$ and drop diameter $D_{0}$ as

$$
\begin{aligned}
& D_{0}{ }^{3} / 6=r_{b 0}{ }^{2} h_{00}, \\
& D_{0}{ }^{3} U_{0}{ }^{2}=b^{2} r_{b 0}{ }^{2} h_{00}\left(3 r_{b 0}{ }^{2}+8 h_{00}\right),
\end{aligned}
$$

where the kinetic energy of the drop after impact is calculated using the velocity distribution in inviscid axisymmetric stagnation point flow: ${ }^{28} w=-2 b z$ and $u=b r$. As the third condition, it is possible ${ }^{23}$ to assume the equality between the preimpact drop velocity and the velocity of the top of the spreading drop $2 b h_{00}$,

$$
U_{0}=2 b h_{00} .
$$

As a result, we obtain from Eqs. (55)-(57)

$$
\begin{aligned}
& b=2^{2 / 3} U_{0} / D_{0}, \\
& h_{00}=2^{-5 / 3} D_{0}, \\
& r_{b 0}=2^{1 / 3} 3^{-1 / 2} D_{0}, \\
& \mathrm{We}_{*}=2^{1 / 3} \frac{\mathrm{We}}{3(1-\cos \theta)} .
\end{aligned}
$$
come

As $\mathrm{We} \rightarrow \infty$, the initial conditions given by Eq. (52) be-

$$
\bar{r}_{b}=0, \frac{d \bar{r}_{b}}{d \bar{\tau}}=1 \text { at } \bar{\tau}=0 .
$$

Thus, for $\mathrm{We} \rightarrow \infty$, the blob motion on the leading edge of a free liquid sheet is described by a universal relationship (independent of the Weber number) for blob coordinates $\bar{r}_{b}$ $=f(\bar{\tau})$, which is a solution of the Cauchy problem, i.e., Eqs. (51) and (62). As for the motion of a blob on the edge of a wall sheet, the advancing stage is described by the same universal relationship that describes blob motion on a free sheet, while the receding stage depends on the advancing and receding contact angles ratio [see Eqs. (53) and (54)]. A transformation from the blob coordinates to the dimensional coordinates $r_{b}, t$ or to the commonly used dimensionless coordinates $\widetilde{r}_{b}=r_{b} D_{0}$ and $\tilde{\tau}=\tau U_{0} / D_{0}$ can be performed by means of Eqs. (49) and (50) accounting for Eqs. (26) and (58)-(60):

$$
\begin{aligned}
& r_{b}=\widetilde{r}_{b} D_{0}, \quad \widetilde{r}_{b}=\bar{r}_{b}\left[\frac{2}{9} \frac{\mathrm{We}}{\left(1-\cos \theta_{1}\right)}\right]^{1 / 2}, \\
& t=\widetilde{t} \frac{D_{0}}{U_{0}}, \quad \tilde{t}=\tilde{\tau}-2^{-2 / 3}, \quad \tilde{\tau}=\bar{\tau}\left[\frac{\mathrm{We}}{6\left(1-\cos \theta_{1}\right)}\right]^{1 / 2} .
\end{aligned}
$$

In the linear approximation, Eqs. (51), (53), (54), and (62) describe free oscillations of a mass-spring system with the variable force constant of a spring in the form of a step function (hereinafter SFM). It is easy to obtain the solution of this problem in the following forms:

$$
\begin{aligned}
& \bar{r}_{b}=\frac{1}{\omega_{a}} \sin \left(\omega_{a} \bar{\tau}\right), \quad \bar{\tau} \in\left[0, \frac{\pi}{2 \omega_{a}}\right], \\
& \bar{r}_{b}=\frac{1}{\omega_{a}} \cos \left(\omega_{r} \bar{\tau}-\varphi\right), \quad \bar{\tau} \geqslant \frac{\pi}{2 \omega_{a}},
\end{aligned}
$$

where $\varphi=\pi \omega_{r} /\left(2 \omega_{a}\right)$.

Using Eqs. (62) and (63) we can evaluate the ratio of the advancing stage time scale $T_{a}$ to the receding one $T_{r}$. Since $\omega_{a}$ and $\omega_{r}$ are angular frequencies at the advancing and receding stages, respectively, we have

$$
\omega_{a} / \omega_{r}=T_{r} / T_{a} .
$$

For example, for the values of $\theta_{1}=92^{\circ}$ and $\theta_{2}=60^{\circ}$ which correspond to the experimental data, ${ }^{4}$ we have $T_{r} / T_{a}$ $=1.44$.

Thus, the foregoing shows that the drop assumes the equilibrium shape in the process of oscillations, which starts the instant the blob speed relative to the wall sheet becomes equal to the velocity of the liquid in the wall sheet. At this instant the edge of the spreading drop comes to a halt and some time later the receding motion starts.

\section{B. Drop oscillation frequency}

This model allows us to evaluate the drop oscillation frequency while it takes its equilibrium shape without finding the exact solution to the problem. Since the relative velocity of the blob over a wall sheet of thickness $h_{00}$ is $\left[\sigma /\left(\rho h_{00}\right)\right]^{1 / 2}$, the oscillation period $T$ can be evaluated as

$$
T=r_{b 0} /\left[\sigma /\left(\rho h_{00}\right)\right]^{1 / 2} .
$$

Then, for the drop oscillation frequency $f=1 / T$ we have

$$
f=\sqrt{\frac{\sigma}{\rho h_{00} r_{b 0}^{2}}} .
$$

Taking into account the condition of the volume conservation given by Eq. (55), Eq. (69) can be rewritten in the following form:

$$
f=\sqrt{\frac{6 \sigma}{\rho D_{0}^{3}}} .
$$

Fukai et al. ${ }^{5}$ carried the numerical simulation of the impact of the molten tin droplet against a solid surface. The parameters of the drop before impact were drop diameter $D_{0}=24 \mu \mathrm{m}$, initial velocity $U_{0}=25 \mathrm{~m} / \mathrm{s}$, surface tension $\sigma$ $=0.554 \mathrm{~N} / \mathrm{m}$, and density $\rho=7000 \mathrm{~kg} / \mathrm{m}^{3}$. According to numerical calculations the frequency of drop oscillations was equal to $1.80 \times 10^{5} \mathrm{~s}^{-1}$, whereas Eq. (70) gives $f=1.85$ $\times 10^{5} \mathrm{~s}^{-1}$.

\section{Evaluation of the drop radius at its maximum extension}

The developed model also allows us to find the instant $\tau_{s}$ when the drop's edge comes to a halt and, correspondingly, to evaluate the maximum radius of the drop without a numerical solution to the problem. To accomplish that, we should note that shortly after the spreading drop's edge has 
halted, the backward motion of the blob begins. This means that at the instant $\tau_{s}$ the function $r_{b}(\tau)$ has a maximum, i.e., $d r_{b}(\tau) / d \tau=0$ at $\tau=\tau_{s}$. Assuming that Eq. (46) is valid for any instant of time and neglecting the change in the blob's mass, we obtain

$$
b r_{b 0}=\left[\frac{\sigma\left(1-\cos \theta_{1}\right)}{\rho h\left(\tau_{s}\right)}\right]^{1 / 2} .
$$

By virtue of Eq. (27), Eq. (71) takes the form

$$
\bar{\tau}_{s}=1,
$$

or, for the dimensionless time $\tilde{\tau}=\tau U_{0} / D_{0}$

$$
\tilde{\tau}_{s}=\sqrt{\frac{\mathrm{We}}{6\left(1-\cos \theta_{1}\right)}} .
$$

The blob's coordinate at the instant $\tau_{s}$ is estimated by integrating the following equation over the time interval $\left[1 / b, \tau_{s}\right]$ :

$$
\frac{d r_{b}}{d \tau}=b r_{b 0}-\left[\frac{\sigma\left(1-\cos \theta_{1}\right)}{\rho h(\tau)}\right]^{1 / 2} \text {. }
$$

As a result we obtain the dimensionless radius of the drop at its maximum extension

$$
\bar{r}_{b s}=\bar{r}_{b}\left(\bar{\tau}_{s}\right)=\frac{1}{2},
$$

or in the dimensionless form related to the preimpact drop diameter $D_{0}$

$$
\tilde{r}_{b s}=\frac{r_{b s}}{D_{0}}=\sqrt{\frac{\mathrm{We}}{18\left(1-\cos \theta_{1}\right)}} .
$$

\section{NUMERICAL METHOD OF SOLUTION AND RESULTS}

To solve the initial boundary problem given by Eqs. (51), (53), (54), and (62), we reduce it to the integral equations by means of twofold integration of Eqs. (51) and (53) accounting for initial boundary conditions, Eq. (62):

$$
f-(f)_{\bar{\tau}=0}-\left(\frac{d}{d \bar{\tau}} f\right)_{\bar{\tau}=0} \bar{\tau}+\omega^{2} \int_{\xi}^{\bar{\tau}} \int_{\xi}^{t} \bar{r}_{b} d t=0, f \equiv \bar{r}_{b}-\frac{\bar{r}_{b}^{3}}{3 \bar{\tau}^{2}},
$$

where $\omega=\omega_{a}$ for the advancing stage and $\omega=\omega_{r}$ for the receding one.

To find the values of second and third terms on the left side of Eq. (77) let us notice that, by virtue of the initial conditions given by Eq. (62), an expansion of the function $\overline{r_{b}}$ in the Maclaurin series has the form

$$
\bar{r}_{b}=\bar{\tau}+O\left(\bar{\tau}^{2}\right) .
$$

Substituting Eq. (78) in Eq. (77) and accounting for the identity $\int_{\xi}^{t} \int_{\xi}^{\tau} f(\tau) d \tau=\int_{\xi}^{t}(t-\tau) f(\tau) d \tau$, we arrive at the integral equation describing the blob dynamics:

$$
\bar{r}_{b}-\frac{\bar{r}_{b}^{3}}{3 \bar{\tau}^{2}}-\frac{2}{3} \bar{\tau}+\omega^{2} \int_{0}^{\bar{\tau}}(\bar{\tau}-\zeta) \bar{r}_{b} d \zeta=0 .
$$

It is significant to recall that, for the case of blob formation at the edge of a free liquid sheet, Eq. (79) describes both advancing and receding stages since the angle $\theta$ keeps a constant value of $180^{\circ}$ and $\omega=2^{1 / 2}$. In the case of spreading over a solid surface Eq. (79) describes only the advancing stage for any value of the advancing angle and $\omega=2^{1 / 2}$.

Introducing the time grid $\bar{\tau}_{i}=\bar{\tau}_{i-1}+\Delta \bar{\tau}$ we can approximate the integral from Eq. (79) at a point $\bar{\tau}_{i}$ by the following finite-difference approximation:

$$
\int_{0}^{\bar{\tau}}(\bar{\tau}-\zeta) \bar{r}_{b} d \zeta \cong \Delta \eta \sum_{k=1}^{i} \frac{\delta_{k}+\delta_{k-1}}{2}\left(\eta_{i}-\frac{\eta_{k}+\eta_{k-1}}{2}\right) .
$$

Here $\delta_{i} \equiv \bar{r}_{b}\left(\eta_{i}\right), \eta_{i} \equiv \bar{\tau}_{i}$, and $\Delta \eta=\Delta \bar{\tau}$.

Accounting for Eq. (80) the finite-difference scheme for Eq. (79) takes the form, at the point $\eta_{i}$,

$$
\begin{aligned}
\delta_{i}^{3}- & 3 \eta_{i}^{2}\left(1+\frac{\Delta \eta^{2}}{2}\right) \delta_{i}+2 \eta_{i}^{3}-6 \eta_{i}^{2} \Delta \eta\left[\frac{\delta_{i-1} \Delta \eta}{4}\right. \\
& \left.+\sum_{k=1}^{i-1} \frac{\delta_{k}+\delta_{k-1}}{2}\left(\eta_{i}-\frac{\eta_{k}+\eta_{k-1}}{2}\right)\right]=0 .
\end{aligned}
$$

Similarly we can derive the integral equation from Eqs. (53) and (54), describing the receding stage of the drop spreading over a solid surface:

$$
\begin{aligned}
f- & (f)_{\bar{\tau}=\bar{\tau}_{\max }}-(d f / d \bar{\tau})_{\bar{\tau}=\bar{\tau}_{\max }}\left(\bar{\tau}-\bar{\tau}_{\max }\right)+\omega_{r}^{2} \int_{\bar{\tau}_{\max }}^{\bar{\tau}}(\bar{\tau}-\zeta) \bar{r}_{b} d \zeta \\
& =0, f \equiv \bar{r}_{b}-\frac{\bar{r}_{b}^{3}}{3 \bar{\tau}^{2}} .
\end{aligned}
$$

Here $\bar{\tau}_{\max }$ is the instant when the drop edge (blob) comes to a halt, that is, it has reached its maximum value.

In the process of solving Eq. (81), it has been found that the equation has three real roots at each time step, two of them are always positive and one is negative. Selection of the smaller positive root or the larger one at each time step gives us the curve $1 a$ or an indefinitely increasing curve 2 in Fig. 4, respectively. From the problem statement it is obvious that only the first solution, which is given by curve $1 a$ has a physical meaning. The point $A$ on the curve $\overline{r_{b}}=f(\bar{\tau})$, at which the condition $d \overline{r_{b}} / d \bar{\tau}=0$ holds, separates the advancing and receding stages. From this solution it follows that the coordinates of point $A$ are $(0.96,0.46)$. That agrees well with the prediction given by Eqs. (72) and (75) [point $B(1,0.5)$ in Fig. 4]. Accounting for the coordinates of point $A$, the finitedifference approximation of Eq. (82) takes a form

$$
\begin{aligned}
\delta_{i}^{3}- & 3 \eta_{i}^{2}\left(1+\omega_{r}^{2} \frac{\Delta \eta^{2}}{4}\right) \delta_{i}+\left[1.272+0.222\left(\eta_{i}-0.96\right)\right] \eta_{i}^{2} \\
& -3 \eta_{i}^{2} \omega_{r}^{2} \Delta \eta\left[\frac{\Delta \eta}{4} \delta_{i-1}+\sum_{k=1}^{i-1} \frac{\delta_{k}+\delta_{k-1}}{2}\right. \\
& \left.\times\left(\eta_{i}-\frac{\eta_{k}+\eta_{k-1}}{2}\right)\right]=0 .
\end{aligned}
$$

The results of numerical solution are presented in Fig. 4. In all cases, the instantaneous radius of spreading drop initially 


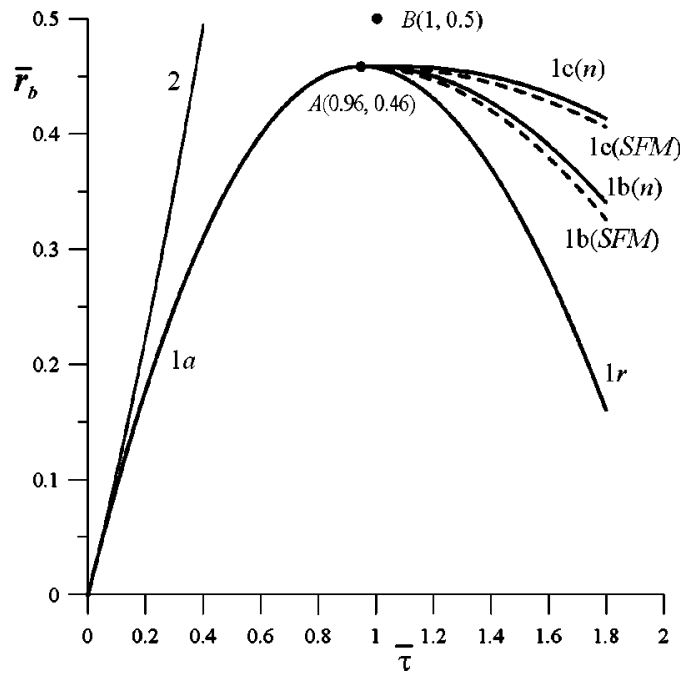

FIG. 4. Universal dependence of the blob coordinate vs time as solution of the Cauchy problem Eqs. (47) and (56). Curves 1 and 2 are the solution branches; point $(0,0)$ is the branching point; point $A$ separates the advancing (curve $1 a$ ) and receding stages (curves $1 r, 1 b, 1 c$ ) of the blob motion; point $B$ is the prediction according to Eqs. (63) and (66). Curve $1 r$ describes the receding stage when condition $\theta_{1}=\theta_{2}$ holds (the blob at the end of a free sheet); curves $1 b$ and $1 c$ describe the receding stage at $\theta_{1}=92^{\circ}, \theta_{2}=60^{\circ}$ and at $\theta_{1}=150^{\circ}, \theta_{2}=40^{\circ}$, respectively. Dash lines-calculation according to SFM [Eqs. (59) and (60)]; solid lines-nonlinear model of the blob motion.

increases with time, then achieves a maximum, and begins to recede. Curve $1 a$ represents the advancing stage of the blob motion, while curve 2 represents the indefinitely increasing branch of the solution. Curve $1 r$ represents the receding stage of blob motion on a free liquid sheet. Curves $1 b(n)$ and $1 c(n)$ represent the receding stages of blob motion on a wall sheet at $\theta_{1}=92^{\circ}, \theta_{2}=60^{\circ}$ and $\theta_{1}=140^{\circ}, \theta_{2}=40^{\circ}$, respectively. The curves $1 b(\mathrm{SFM})$ and $1 c(\mathrm{SFM})$ are the calculations according to SFM for these two regimes of the receding stage. Figure 4 shows that predictions of SFM agree well with calculations according to the nonlinear model of the blob motion and they could be used as a rough estimate of the time scales for advancing and receding stages. For example, for $\theta_{1}=92^{\circ}, \theta_{2}=60^{\circ}$ the SFM model gives the ratio $T_{r} / T_{a}$ $=1.44$, while the nonlinear model gives $T_{r} / T_{a}=1.61$. Substituting values of coordinates of point $A(0.96,0.46)$ into Eqs. (63) and (64) gives the following expressions for the maximum spread diameter, $\xi_{\max }=2 \widetilde{r}_{b s}$, and the dimensionless time $\widetilde{\tau}_{\max }$, required for the drop to reach its maximum extent:

$$
\begin{aligned}
& \xi_{\max }=0.92\left[\frac{2}{9} \frac{\mathrm{We}}{\left(1-\cos \theta_{1}\right)}\right]^{1 / 2}, \\
& \tilde{\tau}_{\max }=0.96\left[\frac{\mathrm{We}}{6\left(1-\cos \theta_{2}\right)}\right]^{1 / 2} .
\end{aligned}
$$

The solutions given by Eqs. (84) and (85) make it possible to evaluate the region where an inviscid approximation holds. To this end, let us note that from the drop mass balance and Eq. (84), it follows that the dimensionless thickness of drop $h^{*}=h / D_{0} \sim \mathrm{We}^{-1}$ at the instant of the maximum drop extension. The dimensionless thickness of the boundary layer can be estimated from the solution for axially symmetrical stag-

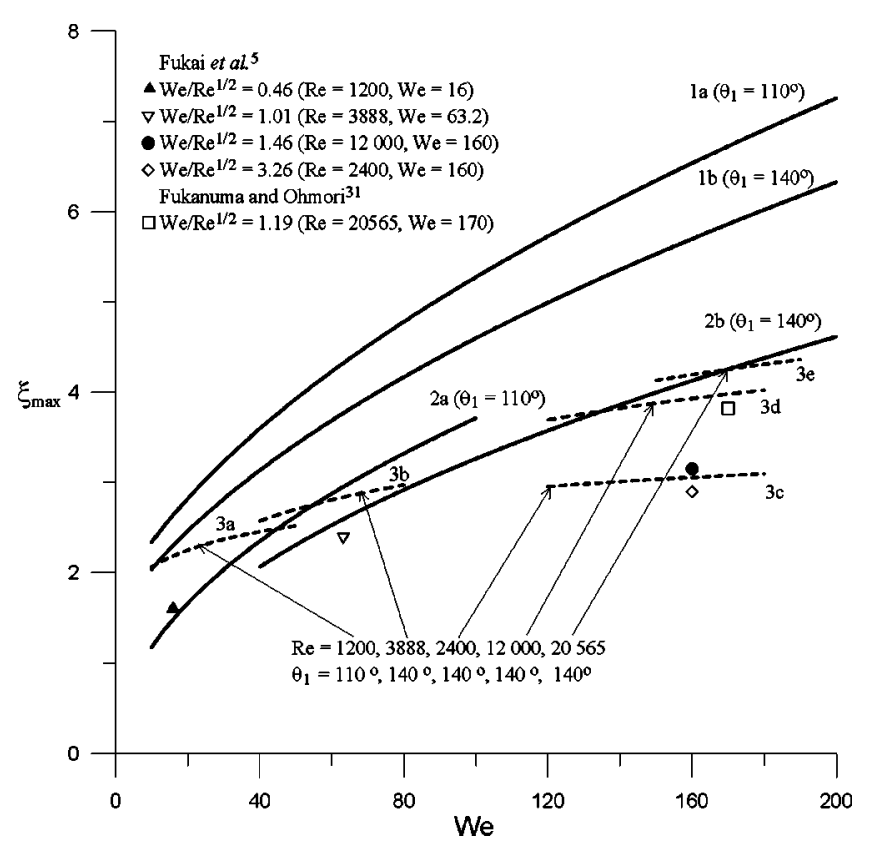

FIG. 5. Comparison analysis of the effect of the Weber number on the dimensionless maximum drop spread diameter $\xi_{\max }$ for different values of $\mathrm{We} / \mathrm{Re}^{1 / 2}$. Solid lines $1 a$ and $1 b$ [from Eq. (88)] and $2 a$ and $2 b$ [from Eq. (84)] represent the variation of $\xi_{\max }$ with the Weber number for $\theta_{1}$ equal to $110^{\circ}$ and $140^{\circ}$, respectively; dashed lines in $3 a-3 e$ present the viscous solution (Ref. 19) given by Eq. (87) for different values of the Reynolds number and for the assumed $\theta_{1}$ values equal to $110^{\circ}$ and $140^{\circ}$.

nation flow ${ }^{28}$ as $\delta^{*}=\delta / D_{0}=\mathrm{Re}^{-1 / 2}$. Solving the inequality $\delta^{*} \ll h^{*}$ yields

$$
\operatorname{Re}^{1 / 2} \gg \mathrm{We} \text {. }
$$

However, it should be borne in mind that this inequality serves only as order of magnitude estimate for the relative influence of capillary and viscous forces on the drop spreading. The actual boundary of the region must be established by comparison with independent calculations or experiments. To this end, predictions of $\xi_{\max }$ obtained from different approaches, namely, the drop energy balance, ${ }^{19}$ numerical solution of the Navier-Stokes equations, ${ }^{5}$ and the present dynamical consideration [Eq. (84)] are compared in Fig. 5.

Here, it is worth noting that expression for the maximum spread diameter $\xi_{\max }$, derived in study ${ }^{19}$ on the basis of the energy balance of impacting drop and the assumption that the drop kinetic energy is zero when the drop is at its maximum extension, is given by the following formula:

$$
\xi_{\max }=\sqrt{\frac{\mathrm{We}+12}{3\left(1-\cos \theta_{1}\right)+4(\mathrm{We} / \sqrt{\mathrm{Re}})}} .
$$

Equation (87) reduces to

$$
\xi_{\max }=\left[(\mathrm{We}+12) / 3\left(1-\cos \theta_{1}\right)\right]^{1 / 2}
$$

if the inequality given by Eq. (86) holds.

Four points in Fig. 5 represent numerical predictions ${ }^{5}$ for the isothermal viscous spreading of a liquid-tin drop impinging on a flat surface at different Re numbers. One measured value of $\xi_{\max }$ from Fukanuma and Ohmori ${ }^{29}$ has been also presented. Figure 5 shows that the predictions from Eq. (84) (curves $2 a$ and $2 b$ ) agree well with numerical solutions ${ }^{5}$ for 
$\mathrm{We} / \mathrm{Re}^{1 / 2}$ equal to $0.46,1.01$, and with the experiment for $\mathrm{We} / \mathrm{Re}^{1 / 2}=1.19$. At higher value of the Reynolds number $\left(\mathrm{Re}=20565\right.$ and $\left.\mathrm{We} / \mathrm{Re}^{1 / 2}=1.19\right)$ the prediction from $\mathrm{Eq}$. (87) (curve $3 e$ ) practically coincides with the present inviscid prediction, i.e., curve $2 b$. Both lines lie close to the measured $\xi_{\max }$ value. At $\mathrm{We} / \mathrm{Re}^{1 / 2}=1.46$ and $\mathrm{Re}=12000$ the present prediction (curve $2 b$ ) is still close to the solution given by Eq. (87) (curve 3d), but their deviation from the numerical result increases. At $\mathrm{We} / \mathrm{Re}^{1 / 2}=3.26$ and $\mathrm{Re}=2400$, the viscous solution from Eq. (87) (curve 3c) agrees well with the numerical result, whereas Eq. (84) gives the substantially overestimated value of $\xi_{\max }$. As to inviscid prediction from Eq. (88) (curves $1 a$ and $1 b$ ), it gives essentially overestimated values of $\xi_{\max }$ in comparison with all other approaches over the whole considered range of $\mathrm{We} / \mathrm{Re}^{1 / 2}$ and $\theta_{1}$ values.

From the aforesaid, the following conclusions can be drawn. There is a quantitative agreement between the predictions from Eq. (84) and numerical computations in the range of $\mathrm{We} / \mathrm{Re}^{1 / 2} \leqslant 1$. Solutions ${ }^{18,19}$ derived on the basis of assumption that the drop kinetic energy vanishes at the instant of the drop maximum extension overpredict the values of $\xi_{\max }$ in both inviscid and viscous cases if $\mathrm{We} / \mathrm{Re}^{1 / 2} \leqslant 1$. It is therefore sure that this consequence comes from the assumption of vanishing of the drop kinetic energy. As follows from Eq. (46), the drop kinetic energy remains generally greater than zero at the instant of the drop maximum extension. Viscous effects have to be taken into account if $\mathrm{We} / \mathrm{Re}^{1 / 2}>1$.

In the case when the capillary wave propagates along the free sheet, away from the blob, the obtained results allow us to derive the following important relationship. To this end notice that since the first wave adjacent to the blob (see Fig. 6) does not leave the blob, it follows that the blob speed $U_{b}$ relative to the liquid in sheet is equal to the phase speed of capillary wave $c$. The phase speed $c$ is readily computed by the linear theory for a capillary wave on a thin sheet ${ }^{27,30}$

$$
c=\sqrt{\frac{\sigma k}{\rho} \tanh \frac{k h}{2}},
$$

where $k=2 \pi / \lambda$ is the instantaneous wave number, $\lambda$ is the instantaneous wavelength, and $h$ is the instantaneous thickness of the sheet.

Equating Eq. (89) to the blob speed $[2 \sigma /(\rho h)]^{1 / 2}$, we arrive at the following transcendental equation:

$$
(\pi / \lambda *) \tanh (\pi / \lambda *)=1,
$$

the root of which is

$$
\lambda *=\lambda / h=2.619 \text {. }
$$

Since it is not difficult to take the measurements of the instantaneous capillary wavelength $\lambda$, the relationship given by Eq. (91) can be used to determine the instantaneous thickness of free sheets experimentally. Figure 6 gives the examples of a determination of instantaneous crown thickness at drop impact on the shallow liquid layer. The measured values of $\lambda$ are $0.5 \mathrm{~mm}$ at $t=10 \mathrm{~ms}$ and $0.93 \mathrm{~mm}$ at $t$ $=20 \mathrm{~ms}$. According to Eq. (91), the instantaneous thickness of the crown is $0.19 \mathrm{~mm}$ at $t=10 \mathrm{~ms}$ and $0.36 \mathrm{~mm}$ at $20 \mathrm{~ms}$.

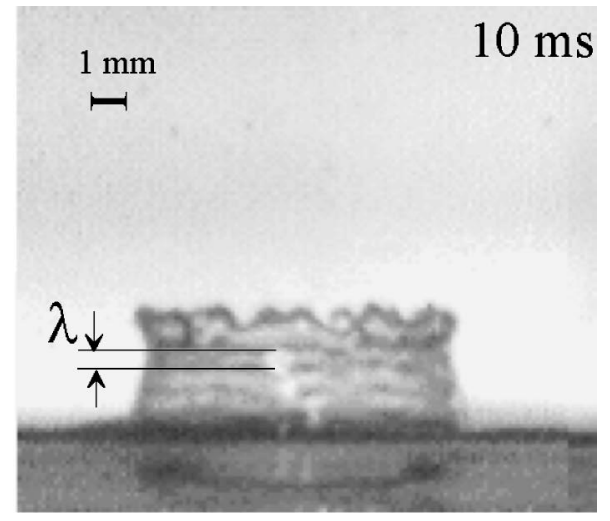

(a)

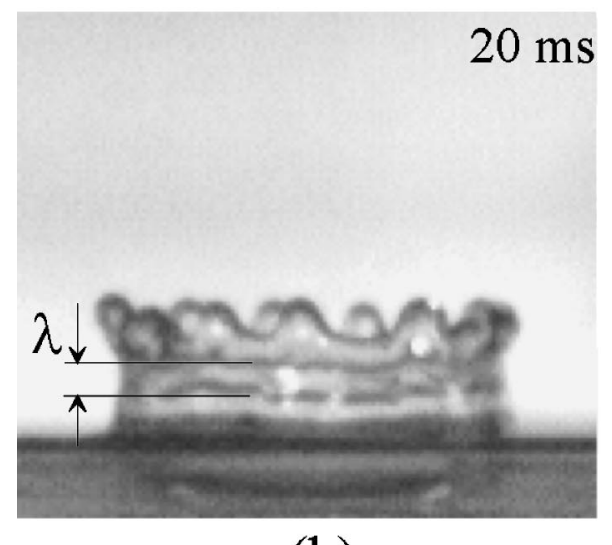

(b)

FIG. 6. Determination of the instantaneous thickness of crown emerging at the drop impact on a shallow water layer of the dimensionless thickness $H^{*}=0.5$. The impact conditions are $U_{0}=2.38 \mathrm{~m} / \mathrm{s}, D_{0}=3.65 \mathrm{~mm}$. Capillary wave with the instantaneous wavelength $\lambda$ propagates along the crown, away from the blob. The instantaneous thickness of the crown $h$ according to Eq. (86) is (a) $h=0.19 \mathrm{~mm}$, (b) $h=0.36 \mathrm{~mm}$.

\section{CONCLUSION}

A physical and mathematical model is proposed for the formation of thick borders on the edge of free and wall sheets induced by drop's impact on liquid and solid surfaces. Based on the present model, some important conclusions have been drawn. In dimensionless blob coordinates, the instant when the drop edge (blob) stops and the radius of the drop at its maximum extension are constants and equal to 1 and 0.5 , respectively. The value of maximum blob coordinate increases as the advancing contact angle $\theta_{a}$ decreases. The developed model has revealed an essential influence of the advancing contact angle on the maximum drop extent and dynamics of the blob formation and motion. Obtained results agree well with theoretical and experimental results ${ }^{4,5,11,18,19}$ both qualitatively and quantitatively. The viscous effects can be neglected during the fast phase of drop spreading if $\mathrm{We} / \mathrm{Re}^{1 / 2} \leqslant 1$.

A linear model based on free oscillation of a mass-spring system with variable spring constant in the form of a step function is suggested to approximate the nonlinear problem. This model makes it possible to determine the frequency of the drop's oscillations without directly solving the nonlinear 
equations of this problem. The solutions obtained from this linear model agree well with those of the nonlinear problem. In the spreading process, the drop reaches its maximum radius at the instant when the relative velocity of the blob becomes equal to the liquid sheet velocity and the overall kinetic energy of the drop remains greater than zero.

Since it is not difficult to take the measurements of the instantaneous capillary wavelength $\lambda$, the relationship given by Eq. (91) can be used to determine the instantaneous thickness of free sheets from experiments. Figure 6 gives the examples of a determination of instantaneous crown thickness at drop impact on the shallow liquid layer.

\section{ACKNOWLEDGMENTS}

This work was supported by the National Science Council, Taiwan, Republic of China under Grant No. NSC912212-E-002-096. The assistance of Y.-H. Wang in numerical calculations is gratefully acknowledged. The valuable comments by Professor K.-C. Wu are also cordially thanked.

${ }^{1}$ A. H. Lefebvre, Atomization and Spray (Taylor \& Francis, London, 1989), pp. 1-34.

${ }^{2}$ A. M. Worthington, "On the forms assumed by drops of liquids falling vertically on a horizontal plate," Proc. R. Soc. London 25, 261 (1876).

${ }^{3}$ A. M. Worthington, A Study of Splashes (Longmans, Green and Co., London, 1908)

${ }^{4}$ J. Fukai, Y. Shiiba, T. Yamamoto, O. Miyatake, D. Poulikakos, C. M. Megaridis, and Z. Zhao, "Wetting effects on spreading of a liquid droplet colliding with a flat surface: Experiment and modeling," Phys. Fluids 7, 236 (1995)

${ }^{5}$ J. Fukai, Z. Zhao, D. Poulikakos, C. M. Megaridis, and O. Miyatake, "Modeling of the deformation of a droplet impinging upon a flat surface," Phys. Fluids A 5, 2588 (1993).

${ }^{6}$ O. P. Solonenko, A. I. Fedorchenko, V. P. Lyagushkin, A. A. Mihalchenko, A. V. Smirnov, P. Chraska, and B. Kolman, in Plasma Jets in the Development of New Materials Technology, edited by O. P. Solonenko and A. I. Fedorchenko (VSP, Utrecht, The Netherlands, 1990), pp. 299-310.

${ }^{7}$ W. E. Ranz, "Some experiment on the dynamics of liquid films," J. Appl. Phys. 30, 1950 (1959).

${ }^{8}$ A. B. Pandit and J. F. Davidson, "Hydrodynamics of the rupture of thin liquid films," J. Fluid Mech. 212, 11 (1988).

${ }^{9}$ A. L. Yarin and D. A. Weiss, "Impact of drops on solid surfaces: Selfsimilar capillary waves, and splashing as a new type of kinematic discon- tinuity," J. Fluid Mech. 283, 141 (1995).

${ }^{10}$ A.-B. Wang and C.-C. Chen, "Splashing impact of a single drop onto very thin liquid films," Phys. Fluids 12, 2155 (2000).

${ }^{11}$ S. D. Aziz and S. Chandra, "Impact, recoil and splashing of molten metal droplets," Int. J. Heat Mass Transfer 43, 2841 (2000).

${ }^{12}$ Lord Rayleigh, "Some application of photography," Nature (London) 44, 249 (1891)

${ }^{13}$ G. I. Taylor, "The dynamics of thin sheets of fluid. III. Disintegration of fluid sheets," Proc. R. Soc. London A253 313 (1959).

${ }^{14}$ R. E. Rosensweig, Ferrohydrodynamics (Cambridge University Press, Cambridge, 1985).

${ }^{15} \mathrm{H}$. N. Oguz and A. Prosperetti, "Surface-tension effects in the contact of liquid surfaces," J. Fluid Mech. 203, 149 (1989).

${ }^{16}$ F. E. C. Culick, "Comments on a ruptured soap film," J. Appl. Phys. 31, 1128 (1960).

${ }^{17}$ M. Song and G. Tryggvason, "The formation of thick borders on an initially stationary fluid sheet," Phys. Fluids 11, 2487 (1999).

${ }^{18}$ J. Madejski, "Solidification of droplets on a cold substrate," Int. J. Heat Mass Transfer 19, 1009 (1976).

${ }^{19}$ M. Pasandidech-Fard, Y. M. Qiao, S. Chandra, and J. Mostaghimi, "Capillary effects during droplet impact on a solid surface," Phys. Fluids 8, 650 (1996).

${ }^{20}$ E. Lugscheider, Y. Borisov, A. Fedorchenko, V. Schimanovich, and M. H. Van de Voorde, "Physical-chemical aspects of thermal spray process and modeling of protective coating formation," INTAS 94-672 Report, Aachen (1997).

${ }^{21}$ A. I. Fedorchenko, "Effects of capillary perturbations on the dynamics of a droplet spreading over a surface," Russ. J. Eng. Thermophys. 10, 1 (2000).

${ }^{22}$ A. Fedorchenko, in Drop-Surface Interaction, edited by M. Rein (Springer, Wien, 2002), pp. 287-290.

${ }^{23}$ A. I. Fedorchenko, "Melt drop spreading at collision with rigid surface," Schwerpunktprogram der Deutschen Forschungsgemeinschaft 'Anwendungsbezogene Optimierung und Steuerung,' Augsburg, No. 361 (1992).

${ }^{24}$ R. L. Hoffman, "A study of the advancing interface I. Interface shape in liquid-gas systems," J. Colloid Interface Sci. 50, 228 (1975).

${ }^{25}$ O. V. Voinov, "Hydrodynamics of wetting," Fluid Dyn. 11, 714 (1976).

${ }^{26} \mathrm{O}$. V. Voinov, "Asymptote to the free surface of a viscous liquid creeping on a surface and the velocity dependence of the angle of contact," Sov. Phys. Dokl. 23, 891 (1978).

${ }^{27}$ S. V. Alekseenko, V. E. Nakoryakov, and B. G. Pokusaev, Wave Flow of Liquid Films (Begell, New York, 1994).

${ }^{28} \mathrm{H}$. Schlichting, Boundary Layer Theory (McGraw-Hill, New York, 1979).

${ }^{29} \mathrm{H}$. Fukanuma and A. Ohmori, "Behavior of molten droplets impinging on flat surfaces," in Proceedings of the Seventh National Thermal Spray Conference, Boston, MA, June 1994, edited by C. C. Berndt and S. Sampath (ASM International, Materials Park, 1994), pp. 563-568.

${ }^{30}$ G. B. Whitham, Linear and Nonlinear Waves (Wiley, New York, 1974). 